BULLETIN (New Series) OF THE

AMERICAN MATHEMATICAL SOCIETY

Volume 29, Number 1, July 1993

\title{
ADDING HANDLES TO THE HELICOID
}

\author{
DAVID HOFFMAN, FUSHENG WEI, AND HERMANN KARCHER
}

\begin{abstract}
There exist two new embedded minimal surfaces, asymptotic to the helicoid. One is periodic, with quotient (by orientation-preserving translations) of genus one. The other is nonperiodic of genus one.
\end{abstract}

We have constructed two minimal surfaces of theoretical interest. The first is a complete, embedded, singly periodic minimal surface (SPEMS) that is asymptotic to the helicoid, has infinite genus, and whose quotient by translations has genus one. The quotient of the helicoid by translations has genus zero and the helicoid itself is simply connected.

Theorem 1. There exists an embedded singly periodic minimal surface $\mathscr{W}_{1}$, asymptotic to the helicoid and invariant under a translation $T$. The quotient surface $\mathscr{W}_{1} / T$ has genus equal to one and two ends.

$\mathscr{W}_{1}$ contains a vertical axis, as does the helicoid, and $\mathscr{W}_{1} / T$ contains two horizontal lines. The second surface is a complete, properly embedded minimal surface of finite topology with infinite total curvature. It is the first such surface to be found since the helicoid, which was discovered in the eighteenth century. (See Figure 1(a).)

Theorem 2. There exists a complete, properly embedded minimal surface, $\mathscr{H}_{e_{1}}$, of genus-one, whose one end is of helicoidal type.

$\mathscr{H} e_{1}$ contains a vertical line, like the helicoid, and one horizontal line that crosses it. Schwarz reflection in these two lines generates the symmetry group of the surface. (See Figure 1(b).)

\section{History AND CONTEXT}

Except for the plane, the helicoid is the only ruled minimal surface. Its discovery is attributed to Meusnier in 1776; together with the catenoid (Euler, c. 1744) these were the only minimal surfaces explicitly known to eighteenth century mathematics. The next major discovery came from Scherk in the 1830s: multiple families of periodic minimal surfaces, including the famous families of singly and doubly periodic examples that bear his name [11, 15]. That the surfaces in these two families share the same normal mapping is implicit in the

Received by the editors July 1, 1992 and, in revised form, December 6, 1992.

1991 Mathematics Subject Classification. Primary 53A10; Secondary 58E12, 49 Q06.

The first author was supported by the National Science Foundation under grants, DMS-9101903 and DMS-9011083 and by the U.S. Department of Energy under grant DE-FG02-86ER25015 of the Applied Mathematical Science subprogram of the Office of Energy Research. The second author was supported by the U.S. Department of Energy under grant DE-FG02-86ER25015 of the Applied Mathematical Science subprogram of the Office of Energy Research. The third author was partially supported by Sonderforschungsbereich SFB256 at Bonn. 


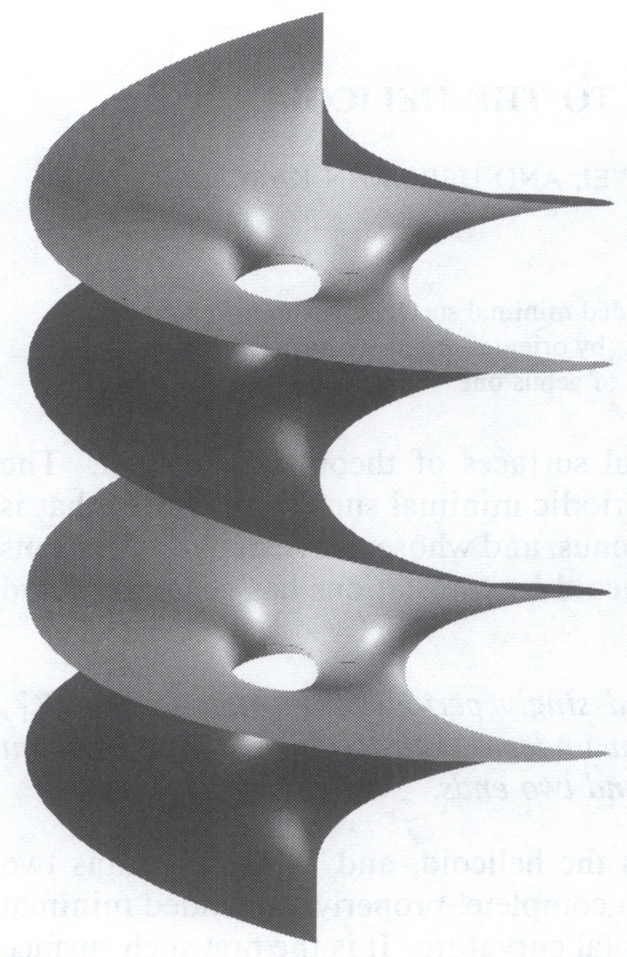

(a)

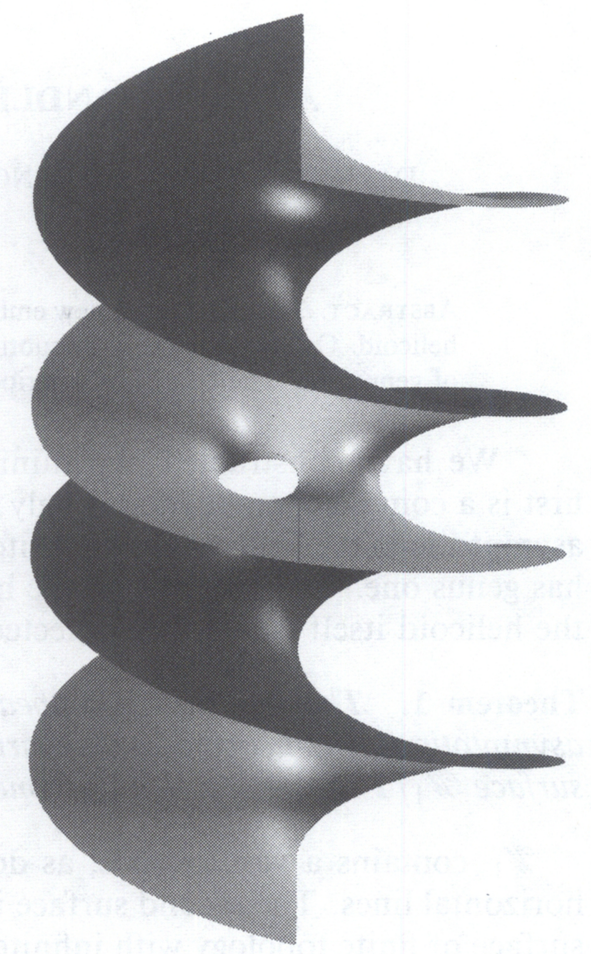

(b)

FIGURE 1. The surfaces: (a) $\mathscr{W}_{1}$ and (b) $\mathscr{H} e_{1}$.

work of Scherk. This fundamental relationship was made explicit by Enneper, Weierstrass, and Riemann. They developed an integral representation formula for minimal surfaces via contour integration of meromorphic data derived from the normal mapping, which they knew to be conformal [13]. (See (3.1) below.) Minimal surfaces were seen to be, from this point of view, the real part of complex curves in $\mathbb{C}^{3}$. The helicoid and catenoid were recognized as, locally, the real and imaginary parts of the same curve. The same is true of the two families of Scherk. Minimal surfaces related in this way are said to be conjugate.

For complete minimal surfaces, whose quotient by orientation-preserving symmetries has finite total curvature, the quotient is naturally a compact Riemann surface, possibly punctured in a finite number of points. Moreover, the meromorphic data is well defined on the compact surface (Osserman [12, 13]). Translations are produced when the integral representation has periods on the Riemann surface. The classical examples mentioned above can be represented on a sphere punctured two (resp. four) times for the helicoid/catenoid pair (resp. Scherk's singly/doubly periodic surfaces). Moreover, the Gauss map can be taken to be the identity on $S^{2}$. All these examples are embedded.

The existence of higher-genus embedded examples has been an open question until recently. For complete embedded examples of finite total curvature (FEMS) in $\mathbb{R}^{3}$, Lopez-Ros [9] showed that the plane and the catenoid are the only FEMS of genus zero. Schoen [16] proved that the catenoid was the only FEMS with two ends. The existence of examples with genus greater than zero 
and more than two ends is well documented [2-5, 18].

The helicoid was the only known example of a complete embedded minimal surface with finite topology and infinite total curvature. It has been a longstanding open question as to whether there are others. Theorem 2 answers this question affirmatively. Also, all known properly embedded examples of infinite total curvature had infinite symmetry groups, and the quotients of these surfaces by these groups were compact (possibly punctured) Riemann surfaces whose inherited metric had finite total curvature. The surface $\mathscr{H} e_{1}$ of Theorem 2 is conformally a once-punctured rhombic torus with symmetry group $Z_{2} \oplus Z_{2}$.

All classical, complete, embedded, doubly periodic minimal surfaces (DPEMS) can be defined by meromorphic data with periods on punctured spheres. Karcher [8] and Meeks-Rosenberg [10] constructed new families of DPEMS that had genus one in the quotient. No higher-genus examples were known that were not coverings of these examples. Moreover, there were no known genus-one examples with the same end behavior as the Scherk doubly periodic examples. In [17] Wei constructed the first DPEMS of genus equal to two in the quotient. Based on the construction strategy used in that paper, Karcher was able to modify Scherk's doubly periodic example to produce a genus-one DPEMS that had the same end behavior as the Scherk example. We refer to this surface as $\mathscr{K}_{\pi / 2}$ for reasons that will be made clear. (See Figure 2.)

\section{SPEMS AS LIMITS OF DPEMS}

The Scherk family can be considered to be the desingularization of two families of equally spaced, parallel, vertical halfplanes meeting at an angle $\theta, 0<\theta \leq \pi / 2$. In the slab $\left|x_{3}\right|<\epsilon$, the surfaces look like saddles over alternating regions in a tiling of $x_{3}=0$ by rhombi. (See Figures 2(a) and 3(a).) With appropriate scaling as $\theta$ goes to zero, the rhombi diagonals grow in one direction only, approaching a strip in the plane. There is a basic relationship between the Scherk family and the helicoid. Namely, if one keeps the symmetric point of a fixed saddle at the origin, the limit surface, with appropriate scaling as $\theta$ goes to zero, exists and is the helicoid (Hoffman and Wohlgemuth [6]).

The generalization, $\mathscr{K}_{\pi / 2}$, of Scherk's surface can be understood as Scherk's surface with a tunnel replacing every other saddle. (See Figure 2(b).) The underlying Riemann surface is the square torus punctured in four points. We proved that this surface can be deformed in exactly the same manner as the Scherk family. (See Figure 3 on page 81 .)

Proposition 1. There exists a one-parameter family $\mathscr{K}_{\theta}$ of embedded doubly periodic minimal surfaces, whose quotient has genus equal to one and four Scherk ends, two up and two down. Each genus-one surface is a rhombic torus. The angle $\theta$ between the up and down ends, $0<\theta \leq \pi / 2$, parametrizes the family.

Each member of the family may be considered to be a desingularization of two families of parallel halfplanes. Unlike the Scherk family, these planes are not equally spaced. The interplanar distance alternates. The smaller distance between planes is spanned by tubes, while the larger one is bridged by saddles. 

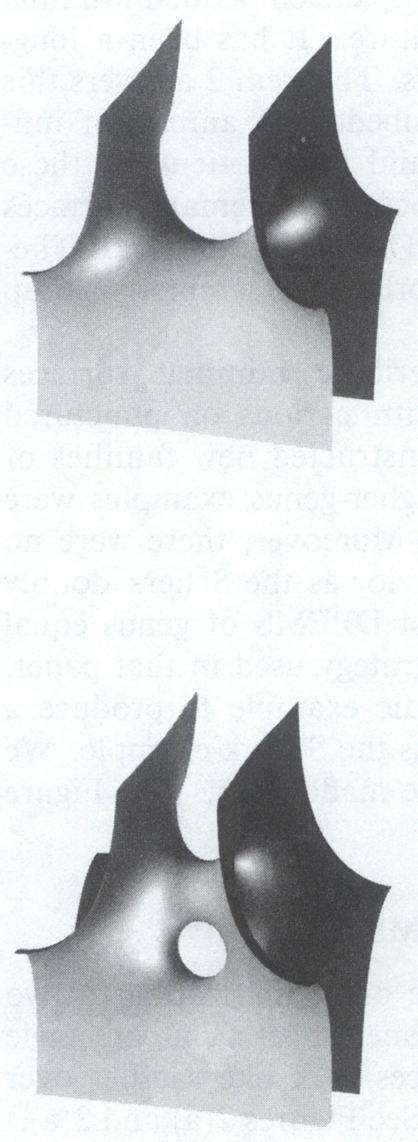

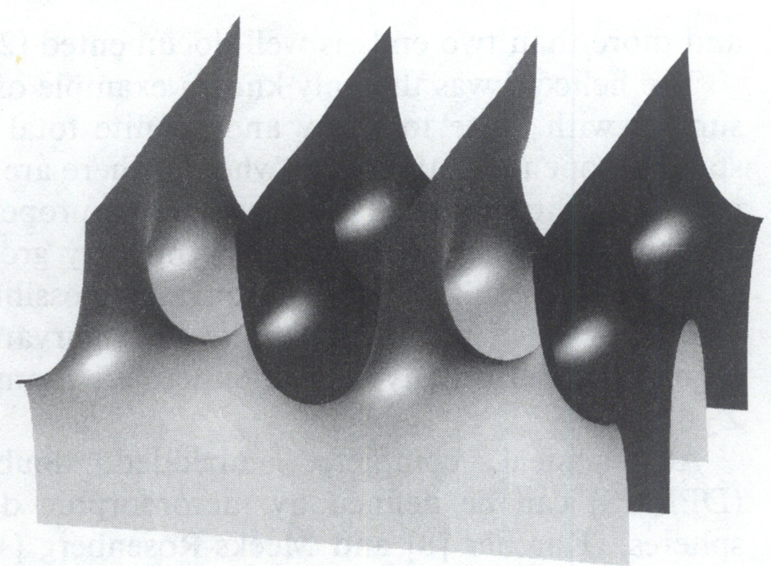

(a)

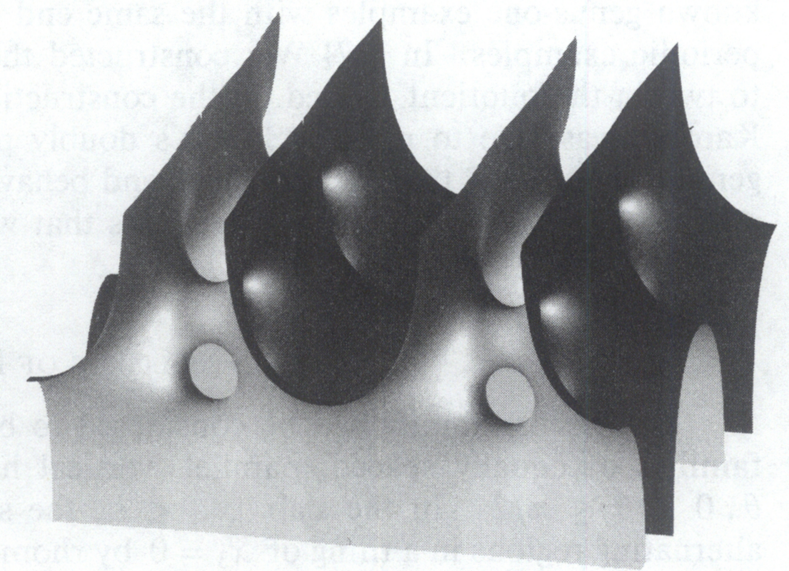

(b)

FIGURE 2. Scherk's doubly periodic surface: (a) $\theta=\pi / 2$ and (b) $\mathscr{K}_{\frac{\pi}{2}}$.

The singly periodic surface $\mathscr{W}_{1}$ of Theorem 1 has the same relationship to $\mathscr{K}_{\theta}$ as the helicoid has to the Scherk family. Namely, choose a distinguished point in a fundamental domain that is identifiable on each surface (e.g., the point on the saddle where the normal is vertical) and keep this point at the origin. Then

Theorem 3. The limit surface as $\theta \rightarrow 0$ of the surfaces $\mathscr{K}_{\theta}$ exists and is equal to $\mathscr{W}_{1}$.

\section{Construction of $\mathscr{H} e_{1}$}

The surface $\mathscr{W}_{1}$ can be described as a helicoid, into which has been sewn a handle at every other half-turn. Thus a handle has been added to the surface modulo translation. One could imagine adding a handle to every other fundamental domain, producing three half-twists between handles, and (why stop at 

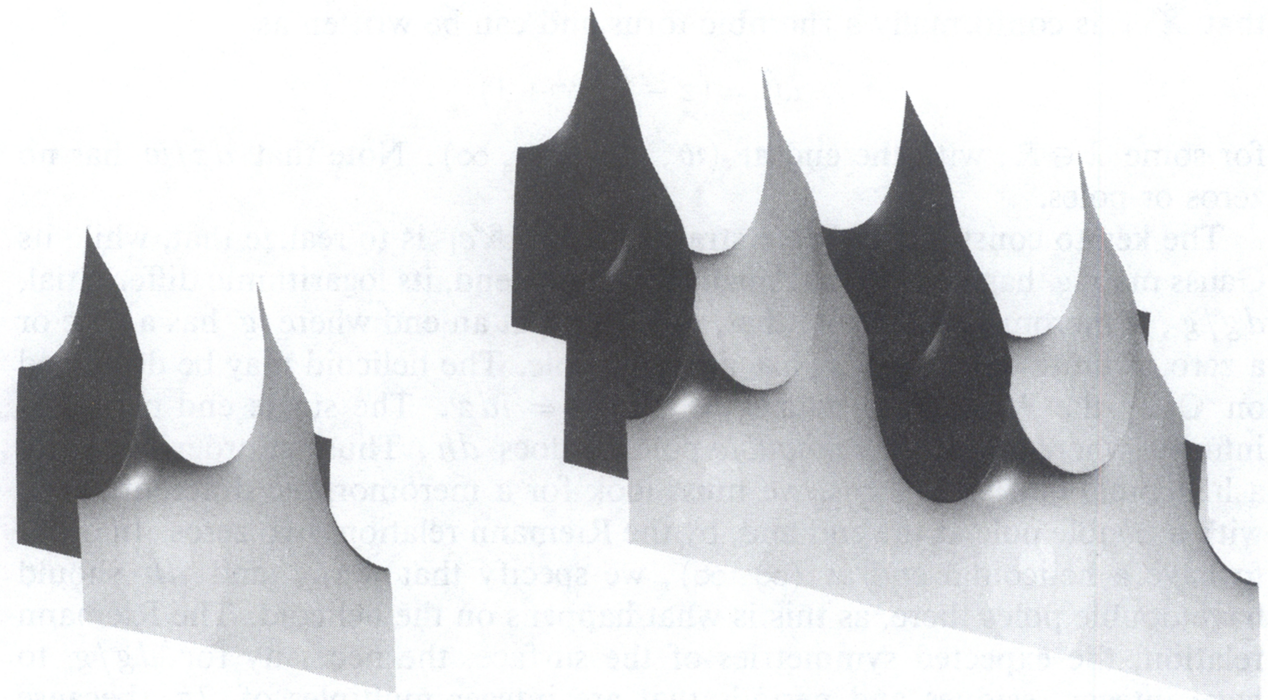

(a)
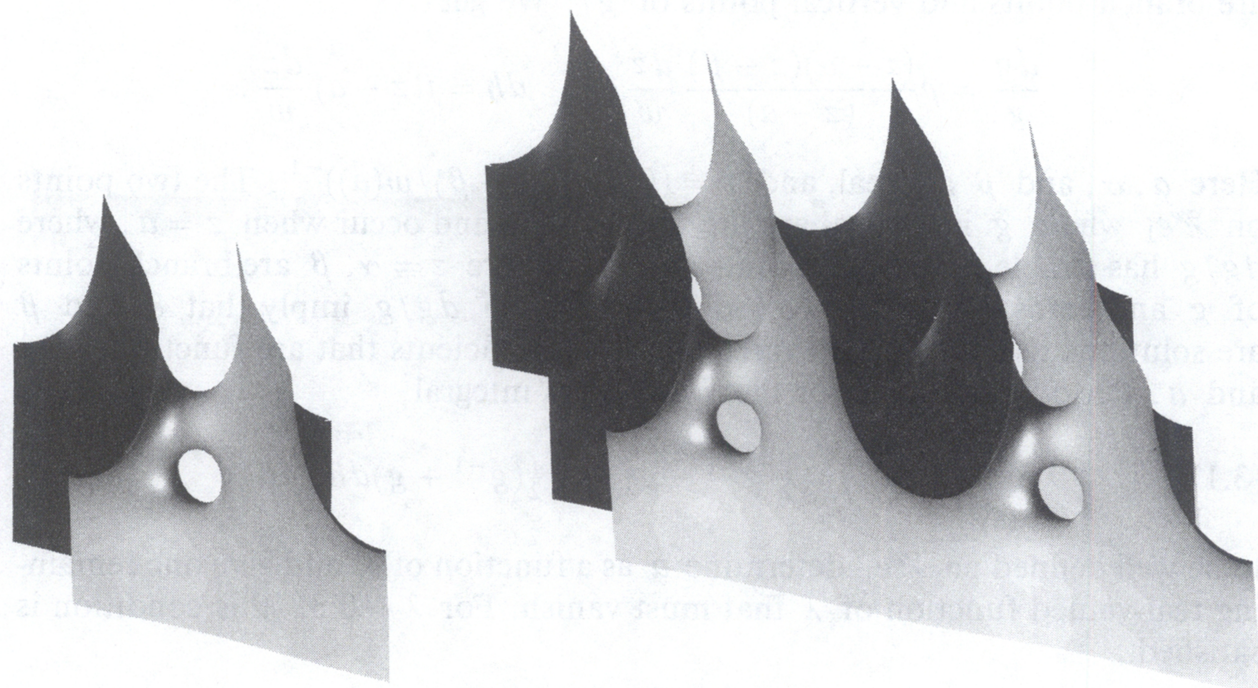

(b)

FIGURE 3. A surface in the Scherk family: (a) $\mathscr{K}_{\theta}$ and (b) $\theta=\pi / 4$.

three?) in general $2 k+1$ half-twists between handles, $k \geq 0$. The quotient by orientation-preserving translations of such a surface will have genus-one. Now imagine fixing one horizontal line in a fundamental domain to be the $x_{2}$-axis and letting $k \rightarrow \infty$. The resulting surface will have genus one, will contain the $x_{2}$-axis and $x_{3}$-axis but no other lines, will not be periodic, and should be asymptotic in some sense to the helicoid. In fact, such a surface exists and is the surface $\mathscr{H} e_{1}$ of Theorem 2 . Because of the presence of two lines on $\mathscr{H} e_{1}$ that cross at one point on the surface (and at the end), $\mathscr{H} e_{1}$ is invariant under two anticonformal reflections with precisely two common fixed points. This implies 
that $\mathscr{H} e_{1}$ is conformally a rhombic torus and can be written as

$$
w^{2}=(z-\lambda)\left(z^{2}+1\right)
$$

for some $\lambda \in \mathbb{R}$, with the end at $(w, z)=(\infty, \infty)$. Note that $d z / w$ has no zeros or poles.

The key to constructing Weierstrass data on $\mathscr{H} e_{1}$ is to realize that, while its Gauss map $g$ has an essential singularity at the end, its logarithmic differential, $d g / g$, is meromorphic. Note that, in general, at an end where $g$ has a pole or a zero of finite order, $d g / g$ has a simple pole. The helicoid may be described on $\mathbb{C}$ by the Weierstrass data $g=e^{z}, d h=i d z$. The single end occurs at infinity, where $d g / g$ has a double pole, as does $d h$. Thus, in order to expect a helicoidal end on a torus, we must look for a meromorphic differential $d h$ with a double pole at the end and, by the Riemann relation, two zeros. In order to have a helicoidal end at $(\infty, \infty)$, we specify that $d g / g$ and $d h$ should have double poles there, as this is what happens on the helicoid. The Riemann relation, the expected symmetries of the surface, the necessity for $d g / g$ to have integer residues and periods that are integer multiples of $2 \pi$ (because $g=e^{\int d g / g}$ must be well defined on $\left.\mathscr{H} e_{1}\right)$, together with the aforementioned Gedanken-experiment, allow us to place the zeros and poles of $d g / g$ (which are branch points and vertical points of $g$ ). We get

$$
\frac{d g}{g}=\rho \frac{(z-\alpha)(z-\beta)}{(z-a)} \frac{d z}{w}, \quad d h=i(z-a) \frac{d z}{w} .
$$

Here $a, \alpha$, and $\beta$ are real, and $\rho=((a-\alpha)(a-\beta) / w(a))^{-1}$. The two points on $\mathscr{H} e_{1}$ where $g$ is vertical are the zeros of $d h$ and occur when $z=a$, where $d g / g$ has simple poles. The points on $\mathscr{H} e_{1}$ where $z=\alpha, \beta$ are branch points of $g$ and zeros of $d g / g$. Period conditions on $d g / g$ imply that $\alpha$ and $\beta$ are solutions to a quadratic expression with coefficients that are functions of $\lambda$ and $a$. Period conditions for the Weierstrass integral

$$
X(p)=\operatorname{Re} \int_{p_{0}}^{p}\left(\frac{1}{2}\left(g^{-1}-g\right) d h, \frac{i}{2}\left(g^{-1}+g\right) d h, d h\right)
$$

to be well defined on $\mathscr{H} e_{1}$ determine $a$ as a function of $\lambda$ and give one remaining real-valued function of $\lambda$ that must vanish. For $\lambda \sim 0.32$ this condition is satisfied.

\section{Computation}

To produce the pictures and find strong experimental evidence that these surfaces exist, MESH [1,7] was used. Computational programs were used to solve the period problems inherent in these representations. A proof for the existence of $\mathscr{W}_{1}$ can be given using a degree theory argument for the period mapping.

\section{ACKNOWledgments}

The authors thank Harold Rosenberg for encouraging us to look for surfaces like $\mathscr{W}_{1}$ and $\mathscr{H} e_{1}$. The program MESH developed at GANG by Jim 
Hoffman et al. was essential to our work; without it we would not have been able to discover this surface $\mathscr{W}_{1}$ by our methods. Hoffman prepared the illustrations for this paper. We were not the first to think about the existence of a minimal surface like $\mathscr{W}_{1}$. In 1985, after viewing images of the helicoid and Costa's surface in a news article [14], Sarah Mellon constructed models of more complicated embedded minimal surfaces of finite total curvature out of wire and strapping tape, and a higher genus helicoid out of clay. She got the finite total curvature surfaces more or less exactly right. The generalized helicoid she constructed was not $\mathscr{W}_{1}$, but the morphology was correct.

\section{REFERENCES}

1. M. Callahan, D. Hoffman, and J. Hoffman, Computer graphics tools for the study of minimal surfaces, Comm. ACM 31 (1988), 648-661.

2. C. Costa, Example of a complete minimal immersion in $\mathbb{R}^{3}$ of genus one and three embedded ends, Bol. Soc. Brasil. Mat. 15 (1984), 47-54.

3. D. Hoffman, The discovery of new embedded minimal surfaces: elliptic functions, symmetry; computer graphics, Proceedings of the Berlin Conference on Global Differential Geometry, Lecture Notes in Math., vol. 1748, Springer-Verlag, New York, 1985.

4. D. Hoffman and W. H. Meeks III, Embedded minimal surfaces of finite topology, Ann. of Math. (2) 131 (1990), 1-34.

5. _ Minimal surfaces based on the catenoid, Amer. Math. Monthly, Special Geometry Issue 97 (1990), 702-730.

6. D. Hoffman and M. Wohlgemuth, Limiting behavior of classical periodic minimal surfaces, GANG preprint series III (to appear).

7. J. T. Hoffman, MESH manual, GANG preprint series II, \#35.

8. H. Karcher, Embedded minimal surfaces derived from Scherk's examples, Manuscripta Math. 62 (1988), 83-114.

9. F. J. Lopez and A. Ros, On embedded complete minimal surfaces of genus zero, J. Differential Geom. 33 (1991), 293-300.

10. W. H. Meeks III and H. Rosenberg, The global theory of doubly periodic minimal surfaces, Invent. Math. 97 (1989), 351-379.

11. J. C. C. Nitsche, Lectures on minimal surfaces, vol.1, Cambridge Univ. Press, Cambridge, 1989.

12. R. Osserman, Global properties of minimal surfaces in $E^{3}$ and $E^{n}$, Ann. of Math. (2) 80 (1964), 340-364.

13. — A survey of minimal surfaces, 2nd ed., Dover, New York, 1986.

14. I. Peterson, Three bites in a doughnut, Sci. News 127 (1985), 168-169.

15. H. F. Scherk, Bemerkungen über die kleinste fläche innerhalb gegebener grenzen, J. Reine Angew. Math. 13 (1835), 185-208.

16. R. Schoen, Uniqueness, symmetry, and embeddedness of minimal surfaces, J. Differential Geom. 18 (1983), 791-809.

17. F. Wei, Some existence and uniqueness theorems for doubly periodic minimal surfaces, Invent. Math. 109 (1992), 113-136.

18. M. Wohlgemuth, Higher genus minimal surfaces by growing handles out of a catenoid, Manuscripta Math. 70 (1991), 397-428. 
Department of Mathematics, University of Massachusetts, AMHerst, Massachusetts 01003

E-mail address: david@gang.umass.edu

Department of Mathematics, Texas A\&M University, College Station, TeXas 77843

Current address: Department of Mathematics, University of Massachusetts, Amherst, Massachusetts 01003

E-mail address: wei@gang.umass.edu

Mathematisches Institut, Universitat Bonn, D-5300 BonN, West Germany

E-mail address: UNM416@IBM.rhrz.uni-bonn.de 